OPEN ACCESS

Edited by:

Donato Traversa,

University of Teramo, Italy

Reviewed by:

Charles D. Mackenzie

Task Force for Global Health,

United States

Susan Catherine Cork,

University of Calgary, Canada

*Correspondence:

Roberto Amerigo Papini roberto.amerigo.papini@unipi.it orcid.org/0000-0002-5928-0663

Specialty section:

This article was submitted to Comparative and Clinical Medicine,

a section of the journal

Frontiers in Veterinary Science

Received: 05 June 2020

Accepted: 13 October 2020

Published: 06 November 2020

Citation:

Papini RA, Lubas G and Sgorbini M

(2020) Incidental Detection of Onchocerca Microfilariae in Donkeys

(Equus asinus) in Italy: Report of Four Cases. Front. Vet. Sci. 7:569916. doi: 10.3389/fvets.2020.569916

\section{Incidental Detection of Onchocerca Microfilariae in Donkeys (Equus asinus) in Italy: Report of Four Cases}

\author{
Roberto Amerigo Papini ${ }^{*}$, George Lubas and Micaela Sgorbini \\ Department of Veterinary Sciences, Veterinary Teaching Hospital "Mario Modenato", University of Pisa, Pisa, Italy
}

This paper reports the occurrence for the first time in Italy of autochthonous Onchocerca infection in donkeys. Four jennies, bred on the same farm, were referred to the Veterinary Teaching Hospital of Pisa for a check-up on ovarian activity $(n=3)$ or for veterinary support during the delivery $(n=1)$. Microfilariae were incidentally detected during the blood smear examination of one jenny. Peripheral blood samples were then collected from the other three jennies and the presence of microfilariae was investigated by Knott's test. Circulating unsheathed microfilariae were identified in all the animals. The level of microfilaraemia was between 1 and 31 microfilariae in $2 \mathrm{~mL}$ of blood. Hematological changes showed moderate eosinophilia in one case or both remarkable eosinophilia and basophilia in another case. Based on molecular findings by PCR and sequencing, the microfilariae showed $98 \%$ sequence similarity with Onchocerca sp. in the NCBI GenBank database (Accession No.: MK541848.1). The present report provides evidence that Onchocerca is an etiological agent of parasitic infection in donkeys in Italy. Our findings highlight the importance of screening in donkeys for Onchocerca even in the absence of clinical indications.

Keywords: microfilariae, Onchocerca, onchocerciasis, donkey, Equus asinus, Italy

\section{INTRODUCTION}

Onchocerca cervicalis, Onchocerca reticulata, and Onchocerca raillieti (Onchocercidae, Spirurida) are filarial thread-like nematodes. O. cervicalis and O. reticulata infect equines worldwide (1). O. cervicalis adult females $(50-70 \mathrm{~cm}$ long) and males $(7-10 \mathrm{~cm}$ long) live in the ligamentum nuchae. O. reticulata adult worms can measure up to $75 \mathrm{~cm}$ (females) or $15-20 \mathrm{~cm}$ (males) and inhabit the flexor tendons and suspensory ligament of the fetlock (2). O. raillietti has only been reported in donkeys in Africa (1) and adult worms live in the ligamentum nuchae (3). They are all viviparous. Adult females release microfilariae (L1) measuring $190-310 \mu \mathrm{m}$ for O. cervicalis, $330-370 \mu \mathrm{m}$ for $O$. reticulata (4), and $180-217 \mu \mathrm{m}$ for O. raillieti (3). When microfilariae are released by the females, they migrate through connective tissues and accumulate in the lymphatics of the upper dermis $(1,2)$. The highest concentrations can be found along the linea alba in the ventral midline, especially near the umbilicus $(1,3,4)$. Microfilariae are also commonly found in the dermis of the face, neck, withers, thorax, and eyes (1). O. raillietti microfilariae have also occasionally been found in the dermis of the head, back, hips, forelimbs, hind limbs, and perineum, though to a limited extent (3). Information is only available for the life cycle of $O$. cervicalis (4), however the transmission pathways of O. cervicalis, O. reticulata, and O. raillieti are likely to be similar. These parasites are all transmitted by blood-sucking midges of Culicoides 
species (1, 2, 4, 5). Mosquitoes (Aedes aegypti) also act as intermediate hosts for O. cervicalis (6). Microfilariae are ingested by insect vectors while feeding on an infected animal. After development within the intermediate host to reach the infective third-stage, L3 larvae are transmitted to other susceptible definitive hosts at the subsequent blood meal (4).

Many studies have been conducted on epidemiological, clinical, diagnostic, pathological, and therapeutic aspects of Onchocerca infection in horses as reviewed by Dagnaw et al. (1). However, with the exception of a relatively recent case report in Italy (7), all these studies are dated. Moreover, only a limited number of studies have investigated the parasitosis in donkeys, almost all of the published studies in donkeys were from African countries, and most focus on $O$. raillieti $(3,5,8-10)$. Currently, little or nothing is known about the current spread of Onchocerca infections in donkeys in Italy. In order to fill this gap, we report a case of autochthonous infection in jennies. We discuss various epidemiological and clinical aspects of the infection in this host species.

\section{MATERIALS AND METHODS}

\section{Case Presentation and Blood Collection}

In June 2019, four adult, pluriparous jennies of the Amiatina breed with ages ranging from 6 to 14 years (median age 13 years) were presented to the Veterinary Teaching Hospital (VTH) of the University of Pisa (geographical coordinates: $43^{\circ} 40^{\prime} 48^{\prime \prime} \mathrm{N}$ $\left.10^{\circ} 20^{\prime} 55^{\prime \prime} \mathrm{E}\right)$. The jennies were from a donkey farm located in central Italy, where animals are used for milk production and reared outdoor in a free animal housing system. On the farm, water ad libitum and food were available in shaded resting spots.

Three out of the four were barren jennies admitted to the VTH for an examination of ovarian activity. The other jenny was pregnant and was diagnosed with rupture of the prepubic tendon before pregnancy, thus this jenny needed veterinary care at the time of delivery.

When admitted to the VTH, jennies were housed in collective paddocks $24 \mathrm{~h}$ a day, fed with meadow hay ad libitum along with commercial equine feed (Equifioc ${ }^{\circledR}$, Molitoria Val di Serchio, Italy) according to the NCR energy recommendations (National Research Council, NRC, 2007), as well as having free access to clean and fresh water.

Upon admission to the VTH, all jennies underwent a complete clinical assessment and coprological examinations by means of a flotation technique with saturated solution of $\mathrm{NaCl}$ (specific gravity $=1.20$ ).

The pregnant jenny showed signs of colic 2 days after delivery and thus underwent further clinical investigations. A complete blood count and biochemistry analyses were also performed. For this purpose, a venous blood sample was drawn via jugular venipuncture and collected in two sterile $10 \mathrm{~mL}$ vacutainer tubes, one with K3-EDTA and one plain tube for serum harvesting. As soon as the blood collection was completed, the sample was labeled (jenny I), immediately transferred to the laboratory of veterinary clinical pathology, and examined. Likewise, a blood work was also carried out in the three barren jennies from the same donkey farm. Blood collection was performed in the same way as previously described. The samples were labeled for animal identification (jennies II-IV).

\section{Blood Analysis}

Complete blood counts were performed using a combined laser-impedance cell counter (ProCyte ${ }^{\circledR}$, Idexx Laboratories, Milan, Italy) including red blood cell count, hematocrit, hemoglobin, mean corpuscular volume, mean corpuscular hemoglobin, mean corpuscular hemoglobin concentration, red blood cell distribution width, total leukocyte count, absolute values of neutrophils, lymphocytes, monocytes, eosinophils, and basophils, platelet count, and mean platelet volume. In order to complete the information on the morphological aspects of red blood cells, white blood cells, and platelets, and to assess the leukocyte differential counts in comparison to the results provided by the cell counter, fresh blood smears were prepared and stained with May-Grundwald-Giemsa (MGG) using an automatic stainer (Wescor Aerospray $7150^{\circledR}$, Delcon, Milan, Italy). Using bright-field light microscopy, blood smears were screened at $20 \times$ and then examined at $100 \times$ by a trained clinical pathologist.

The serum biochemical assays were performed on a combined spectrophotometric-immunoturbidimetric device (Analyzer SAT $450^{\circledR}$, Medical System, Guidonia, Rome, Italy) including: total plasma protein, albumin, gamma glutamyl transferase, aspartate aminotransferase, creatine phosphokinase, blood urea nitrogen, creatinine, calcium, phosphate, total bilirubin, and direct bilirubin. In addition, few electrolytes such as sodium, potassium and chloride were assessed with an ion-selective electrodes device (Electrolyte Analyzer GE200 ${ }^{\circledR}$, Medical System, Guidonia, Rome, Italy).

The blood cell counter was set up to evaluate the parameters as equine species. All the reference intervals (RIs) used were derived from an internal assessment carried out by the veterinary clinical pathology laboratory with multi-year experience in examining donkey blood.

\section{Detection of Circulating Microfilariae}

Since microfilariae were detected through the blood smear examination of jenny II (Figure 1), the occurrence of peripherally-circulating microfilariae was also evaluated in the other three jennies. Microfilariae detection was performed using a modified Knott's test on peripheral EDTA blood samples collected, as described above. Briefly, $8 \mathrm{~mL}$ of distilled water were added to $2 \mathrm{~mL}$ of whole EDTA blood and mixed thoroughly. Following centrifugation at 3,000 rpm for $5 \mathrm{~min}$, the supernatant was discarded. The sediment was re-suspended in two drops of distilled water, removed with a $1 \mathrm{~mL}$ Pasteur pipette, and examined with a light microscope at $100 \times$ magnification. The number of microfilariae was counted in the Knott's test sediment. See the Results section for details on morphometric features. After microscopic examination, another aliquot $(2 \mathrm{~mL})$ of EDTA blood sample from each positive jenny was stored at $-20^{\circ} \mathrm{C}$ prior to shipment to a sequencing service provider. 


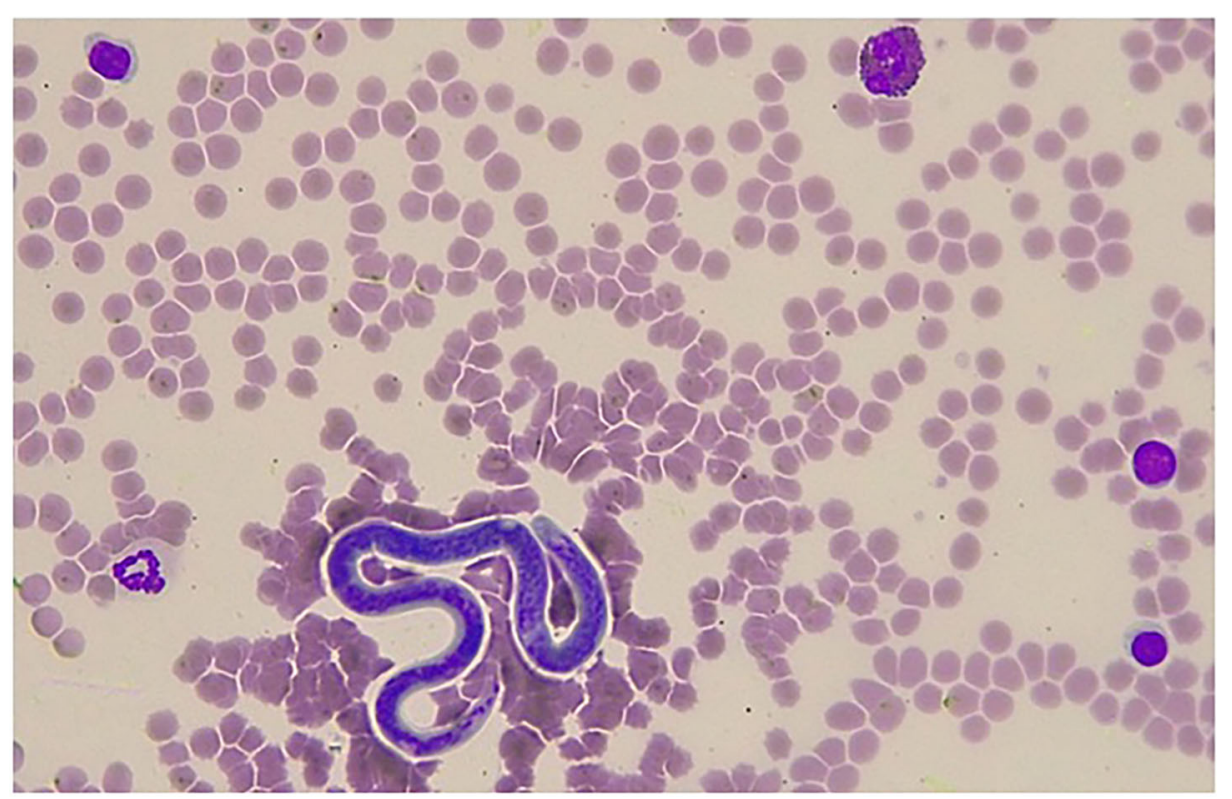

FIGURE 1 | Giemsa stained blood smear showing a single microfilaria of Onchoerca sp. detected in a jenny in Italy. In the microscopic field, one eosinophil with characteristic feature of the asinine species, three lymphocytes, and one neutrophil can also be identified ( $\times 400$ magnification).

\section{Molecular Procedures}

Molecular analyses were performed by BMR Genomics (Padua, Italy). After thawing at room temperature, genomic DNA was extracted from positive blood samples using the commercial DNA IQ ${ }^{\mathrm{TM}}$ System ${ }^{\circledR}$ (Promega) based on paramagnetic beads, following the manufacturer's instructions. A fragment (about 700 base pairs in length) of the mitochondrial gene for cytochrome c oxidase subunit 1 (COX1) was used as a DNA barcoding system and amplified. The PCR amplification was carried out in a final mixture containing $12.5 \mu \mathrm{L}$ of Master Mix 2X $\left(\right.$ GoTaq $^{\circledR}$ Green Master Mix, Promega), $2 \mu \mathrm{L}$ of genomic DNA extracted from blood, $1 \mu \mathrm{L}(10 \mu \mathrm{M})$ of forward primer $\left(5^{\prime}\right.$ TGATTGGTGGTTTTGGTAA-3'), $1 \mu \mathrm{L}(10 \mu \mathrm{M})$ of reverse primer ( $5^{\prime}$-ATAAGTACGAGTATCAATATC-3'), and deionized water, reaching a total reaction volume of $25 \mu \mathrm{L}$. The PCR reactions were subjected to the following conditions in thermal cycler (Mastercycler ${ }^{\circledR}$, Eppendorf): $95^{\circ} \mathrm{C} \times 2 \mathrm{~min}$, then 5 cycles $\left(95^{\circ} \mathrm{C} \times 40 \mathrm{~s}, 45^{\circ} \mathrm{C}\right.$ for $1.5 \mathrm{~min}$, and $72^{\circ} \mathrm{C}$ for $\left.1.5 \mathrm{~min}\right)$, followed by 35 cycles $\left(95^{\circ} \mathrm{C}\right.$ for $40 \mathrm{~s}, 50^{\circ} \mathrm{C}$ for $1.5 \mathrm{~min}$, and $72^{\circ} \mathrm{C}$ for $1 \mathrm{~min}$ ), and finally $72^{\circ} \mathrm{C}$ for $10 \mathrm{~min}$. The amplification products were visualized after electrophoresis on $1.5 \%$ agarose gel. PCR products were purified by AMPure XP Beads (Beckman Coulter), sequenced, and aligned via BLAST analysis to detect their identity by retrieving similar sequences deposited in NCBI's GenBank database $(11,12)$.

\section{Ethics Approval for the Study}

Ethical review was not required as per institutional guidelines/local legislation due to natural infections that occurred spontaneously. Written informed consent was obtained from the owners for the participation of their animals in this study.

\section{RESULTS}

All four jennies were apparently healthy on the basis of history and physical examination at admission to the VTH. Three of them (II, III, and IV) remained in this condition throughout hospitalization. However, one jenny (I) was apparently healthy at admission to the VTH but presented signs of colic 2 days after delivering her foal. The results of the complete blood count for all four jennies are reported in Table 1. In jenny I, the values evidenced an anemic condition with normocytic hyperchromic red blood cells. Hyperchromia was due the lipemic sample collected, which interferes with the correct spectrophotometric reading of hemoglobin and induces a high value of mean corpuscular hemoglobin concentration. In the leukogram, neutrophilia, and monocytosis were found along with lymphopenia, all three conditions could be related to a long-lasting inflammatory process or simply related to stress. In jennies II and III, the values did not reveal anything particular in the red blood cell compartment. In the leukogram, eosinophilia was found in both animals and in jenny III it was substantial as was the occurrence of basophilia. In jenny IV, the results of the complete blood count were all within the RIs.

The biochemistry analysis in jenny I revealed renal insufficiency highlighted by an increase in both urea (48 $\mathrm{mg} / \mathrm{dL}, \mathrm{RI}=9-31)$ and creatinine $(2.2 \mathrm{mg} / \mathrm{dL}, \mathrm{RI}=0.59-1.3)$ as well as a decrease in total proteins $(5.6 \mathrm{~g} / \mathrm{dL}, \mathrm{RI}=5.8-7.6)$ due to a mild reduction in albumin $(2.2 \mathrm{~g} / \mathrm{dL}, \mathrm{RI}=2.5-3.2)$. Both the cholesterol (195 mg/dl, RI $=55-115)$ and triglycerides 
TABLE 1 | Alterations detected in the complete blood count of four jennies found incidentally infected with Onchocerca microfilariae.

\begin{tabular}{|c|c|c|c|c|c|}
\hline \multirow[t]{2}{*}{ Hematological values } & \multirow[b]{2}{*}{$\mathbf{I}$} & \multirow[b]{2}{*}{ II } & \multirow[b]{2}{*}{ III } & \multirow[b]{2}{*}{ IV } & \multirow[t]{2}{*}{ Reference range* $^{*}$} \\
\hline & & & & & \\
\hline Red blood cells $M / \mu L$ & $3.40 \downarrow$ & 6.47 & 5.42 & 5.25 & $4.4-7.1$ \\
\hline Hematocrit \% & 18.5 & 35.2 & 30.9 & 29.8 & $27-42$ \\
\hline Hemoglobin g/dL & $7.1 \downarrow$ & 12.1 & 10.9 & 10.5 & $9.1-14.7$ \\
\hline Mean corpuscular volume fL & 54.4 & 54.4 & 57.0 & 56.8 & $53-67$ \\
\hline Mean corpuscular hemoglobin pg & 20.9 & 18.7 & 20.1 & 20.0 & $17.6-23.1$ \\
\hline Mean corpuscular hemoglobin concentration g/dL & $38.4 \uparrow$ & 34.4 & 35.3 & 35.2 & $32-37.5$ \\
\hline Red blood cell distribution width \% & 21.4 & $22.9 \uparrow$ & 20.5 & 22.4 & $16.1-22.5$ \\
\hline Total leukocytes $\mathrm{K} / \mu \mathrm{L}$ & 14.78 & 10.95 & 15.69 & 6.40 & $6.2-16$ \\
\hline 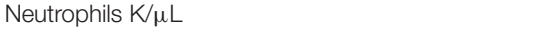 & $11.82 \uparrow$ & 5.04 & 5.49 & 3.07 & $2.4-6.3$ \\
\hline Lymphocytes K/ $\mu \mathrm{L}$ & $1.18 \downarrow$ & 4.27 & 6.12 & 2.28 & $1.6-8.5$ \\
\hline Monocytes $\mathrm{K} / \mu \mathrm{L}$ & $1.77 \uparrow$ & 0.33 & 0.31 & 0.32 & $0.0-0.8$ \\
\hline 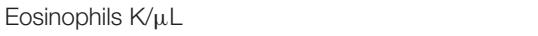 & 0.00 & $1.31 \uparrow$ & $3.45 \uparrow$ & 0.73 & $0.1-1.0$ \\
\hline Basophils $\mathrm{K} / \mu \mathrm{L}$ & 0.00 & 0.00 & $0.31 \uparrow$ & 0.00 & $0.0-0.2$ \\
\hline Platelets $\mathrm{K} / \mu \mathrm{L}$ & 296 & 190 & 295 & 190 & $95-360$ \\
\hline Mean platelet volume fL & 10.4 & 10.0 & 10.4 & 10.1 & $8.8-12.0$ \\
\hline Appearance of plasma & Lipemia ++ & Clear & Clear & Clear & NA \\
\hline Microfilaraemia at the blood smear & 0 & 2 & 0 & 0 & NA \\
\hline Microfilaraemia by Knott's technique & 4 & 31 & 1 & 3 & NA \\
\hline
\end{tabular}

"Reference range used internally at the Clinical Pathology Laboratory; the values out-the-range are in italics; $\downarrow$, decreased values; $\uparrow$, increased values; NA, not applicable.

(663 $\mathrm{mg} / \mathrm{dl}$, RI $=53-248)$ showed high values. All the other parameters tested, namely calcium, phosphate, gamma glutamyl transferase, aspartate aminotransferase, creatine phosphokinase, total bilirubin, and direct bilirubin were within the RIs. In the electrolytes, sodium (125 mEq/L, RI $=128-138)$ and potassium $(1.6 \mathrm{mEq} / \mathrm{L}, \mathrm{RI}=3.2-5.1)$ were low probably due to the interference of the lipemic sample, while chloride was in the normal range. Overall, the trend of these parameters along with the complete blood count results highlighted lipidosis due to a negative energy intake balance related to obstructive colic in a lactating jenny. The clinical symptoms were depression, anorexia, and oliguria (the jenny refused to drink). The mild increase in urea could be related both to anorexia, or along with high levels of creatinine, to an acute pre-renal insufficiency secondary to low ingestion of water. In the other three jennies all parameters on serum biochemistry and electrolytes investigated were within the RIs (values not reported).

In jenny II, microfilaraemia was detected when the blood smear examination by conventional staining and optical microscopy reading was performed by an expert clinical pathologist (Figure 1). On the other hand, no microfilariae were detected through the blood smear evaluation in the other three jennies. MGG stained microfilariae had a wider and rounded anterior end, while the posterior end showed a short, thin, and sharply pointed tail. The microfilariae measured $\sim 214-229 \mu \mathrm{m}$ in length and $4-5 \mu \mathrm{m}$ in width. The most prominent feature was the lack of a faintly stained anterior and posterior sheath. This suggested that on the basis of morphological features, they could be differentiated from the sheathed microfilariae of Setaria spp and belonged to the genus Onchocerca, in which the length of microfilariae between the different species parasitizing donkeys falls within the range of 180-370 $\mu \mathrm{m}(3,4)$.

Since circulating microfilariae were incidentally detected by microscopic examination of a blood smear from one of the jennies, we thus examined the peripheral blood samples from each jenny by a modified Knott's test, as described above. The Knott's test results showed that all the four jennies presented microfilariae in their peripheral blood samples. Circulating microfilariae appeared to be morphologically identical to each other and from one jenny to another. They were actively motile with serpentine movements, showing the lack of a sac-like hyaline sheath at both ends and a short tail, which matched the characteristics exhibited by the microfilariae in MGG stained blood smears. The characteristic features of the microfilariae appeared to be consistent with Onchocerca microfilariae and no further morphometric investigations were carried out, as microfilariae found in Knott's test sediments and in MGG stained smears appeared to be morphologically identical. However, the unusual site of the finding (i.e., peripheral blood stream) precluded any definitive conclusion and only a presumptive diagnosis of asymptomatic onchocerciasis could be made at that time. The levels of microfilaraemia were $31,4,3$, and 1 microfilariae in $2 \mathrm{~mL}$ of blood samples from jennies II, I, IV, and III, respectively. The highest level of microfilaraemia was detected in jenny II which had been found positive from the blood smear. This suggests that positivity at the blood smear was probably related to the higher burden of circulating microfilariae. Table 1 shows the Knott's test results.

The coprological results were positive for gastrointestinal strongyle eggs. 
TABLE 2 | Accession numbers and description of sequences retrieved from GenBank producing significant alignments with COX1 mitochondrial gene of microfilariae incidentally found in peripheral blood samples of four jennies in Italy.

\begin{tabular}{|c|c|c|c|c|}
\hline Accession no. & Parasitic organism identification & Bit score & $E$-value & Max identity \\
\hline MK541848.1 & Onchocerca sp. JJP-2019 & 1,138 & 0.0 & $98 \%$ \\
\hline KX898458.1 & Onchocerca boehmi Supperer, 1934 & 835 & 0.0 & $90 \%$ \\
\hline LC318284.1 & Onchocerca flexuosa & 833 & 0.0 & $90 \%$ \\
\hline AJ271616.1 & Onchocerca gibsoni & 833 & 0.0 & $90 \%$ \\
\hline AM749269.1 & Onchocerca skrjabini & 824 & 0.0 & $91 \%$ \\
\hline AP017692.1 & Onchocerca flexuosa & 817 & 0.0 & $89 \%$ \\
\hline KX265050.1 & Dirofilaria sp. "hongkongensis" & 817 & 0.0 & $89 \%$ \\
\hline AM749270.1 & Onchocerca skrjabini & 813 & 0.0 & $91 \%$ \\
\hline AB518693.1 & Onchocerca sp. wild boar & 808 & 0.0 & $89 \%$ \\
\hline KX853323.1 & Onchocerca boehmi Supperer, 1934 & 806 & 0.0 & $90 \%$ \\
\hline
\end{tabular}

The BLAST search results showed that COX1 of microfilariae from the four examined jennies had the closest sequence similarity (i.e., 98\%) with that of Onchocerca sp. available from GenBank (Accession Number MK541848.1). Sequences producing significant alignments are shown in Table 2.

Unfortunately, no follow-up was possible as the jenny with the ruptured prepubic tendon died after delivery, while the others returned to the farm a few days after check-up on ovarian activity. The owner was unwilling either to take them back to the VHT for further examination or to allow us to visit his farm at a later time.

\section{DISCUSSION}

To the best of our knowledge, this is the first report of Onchocerca infection in donkeys in Italy. The presence of microfilariae in peripheral blood samples was ascertained by laboratory and molecular findings. Unfortunately, the species of microfilariae was not identified. The two Onchocerca species infecting donkeys in Europe are O. cervicalis and O. reticulata, which are both found worldwide (1). The geographical range of $O$. railliet $i$ infection in donkeys known to date is restricted to some countries in Africa $(3,5,8)$. In previous studies, when the occurrence of $O$. cervicalis and $O$. reticulata was investigated concurrently, O. cervicalis was more prevalent than $O$. reticulata. In fact, prevalence values of 82.98 and $4.26 \%$ have been reported for O. cervicalis and $O$. reticulata in donkeys, respectively (13). Similarly, prevalence values of $25.42,5.93$, and $2.54 \%$ have been reported for $O$. cervicalis, O. reticulata, or both infections in horses, respectively (14). Therefore, based on the results of previous surveys, we believe that $O$. cervicalis was most likely the species involved in our study.

All the four Onchocerca infected jennies were bred on the same farm and were kept under the same management conditions. They had always lived on the same farm since their birth and there were no movements throughout the country except for their transportation to the VTH. Onchocerca infection in horses is considered rare when macrocyclic lactones are used regularly $(15)$, since treatments with ivermectin $(16,17)$, moxidectin $(18,19)$ or doramectin (9) are effective in killing microfilariae (but not against adult worms located in the nuchal ligament).
However, since our four jennies were milk-producing animals, no treatment for ectoparasite or endoparasite control had never been performed at the donkey farm in order to prevent the risk of contamination with drug residues in milk intended for human consumption. Moreover, the Culicoides species, which may act as intermediate hosts for Onchocerca, are spread throughout Italy, including central regions (20) where the farm of the infected jennies is located. Therefore, the examined jennies likely acquired the infection locally on the farm and the source of the infection was the bite of infected midges.

There have been few epidemiological studies on Onchocerca infection in donkeys. Reported prevalence values are $65.38 \%$ (21) and $82.98 \%$ (13) for O. cervicalis in Egypt, 34\% for O. raillieti in Sudan (10), and $4.26 \%$ for O. reticulata in again Egypt (13). In another study, only one donkey was examined and found to be infected with $O$. cervicalis (22). No influence of sex on the infection rate has been observed $(5,10,13)$. However, a statistically $(p<0.05)$ higher prevalence has been reported for O. raillieti (64.3\%) in donkeys aged between 7 and 10 years (10) and for O. cervicalis (100\%) in donkeys older than 15 years (10). Similarly, Onchocerca infection has been reported to be more common in older horses rather younger horses (23-25), particularly over 15 years of age $(25,26)$, as the prevalence of infection increases with age (25). Our detection of Onchocerca infection in jennies aged 6-14 years thus appears to be in agreement with the results of previous prevalence studies on donkeys and horses.

The occurrence, distribution, and population density of microfilariae in tissues of Onchocerca infected hosts may vary by season. The peak of distribution of $O$. cervicalis microfilariae in ventral-midline skin of 15 naturally infected pony mares was investigated over a 13-month period and was shown to be highest during the spring and lowest in the winter, disappearing in the surface layers of the dermis during the winter months (27). A distinct pattern of distribution was also reported in blood samples from 284 camels (Camelus dromedarius) where the highest monthly prevalence of Onchocerca microfilariae throughout a period of 14 months was detected in June, disappearing in July to September and February (28). The authors concluded that this is correlated with an adaptation of the parasite to the 
climate, thus affecting the seasonal distribution of the insect vectors (27), and that environmental conditions may arrest the development of microfilariae or influence their distribution in the host's tissues (28). Similar seasonal variations likely occur in the donkey too in terms of the concentration, distribution, and occurrence of microfilariae in tissues, including the bloodstream. Therefore, our finding of Onchocerca microfilariae circulating in the peripheral blood of jennies in late spring (June) is in agreement with the results of other studies $(27,28)$.

Detection of Onchocerca microfilariae in the peripheral blood smear of donkeys or other equids is unusual and thus molecular analysis for confirmatory purposes was needed. In our study, donkeys did not show any dermal or ocular sign that might suggest Onchocerca infection at the time of admittance to the VTH. Therefore, skin biopsies (see the Section Discussion below) were not included in the initial diagnostic workup. On the other hand, onchocerciasis diagnosis with skin biopsy is laborious and time consuming for equine practitioners and laboratory technicians, as well as being expensive for owners. Even when Onchocerca infection was suspected because of microfilariae morphological features, performing skin biopsies of the ventral midline on at least one of the jennies would have required the specific owner's informed consent. Unfortunately, for this veterinary procedure, the jennies need to be sedated to avoid any risk for the operators and also an extended hospitalization time at the $\mathrm{VTH}$, thus leading to economic losses because of the additional costs and lack of income for the owner of a milk production farm. This means that skin biopsies would never have been permitted by the owner given the lack of any clinical evidence and above all with no apparent economic benefit for him. Consequently, we opted to use molecular analysis rather than skin biopsy to confirm a case of microfilaraemia compatible with presumptive laboratory diagnosis of asymptomatic Onchocerca infection. This was despite the fact that a skin biopsy is the standard technique for diagnosing equine onchocerciasis. The concentration of microfilariae that we found in the peripheral blood samples (range 1-31 microfilariae in $2 \mathrm{~mL}$ ) is likely to be much lower than the actual microfilariae concentration in the tissues of the examined jennies because Knott's test is not the most suitable method to detect microfilariae in Onchocerca infected equids. Onchocerca microfilariae have a very uneven distribution in the dermis of their hosts (29). The most effective method to detect Onchocerca microfilariae and accurately estimate their concentration is based on skin biopsies of the midline of the abdomen, preferably near the umbilical region, both in infected donkeys $(5,10)$ and horses $(15,30-32)$. For instance, Hussein and El Sammani (5) reported that the density of $O$. raillieti microfilariae in donkeys ranged from 1,200 to 26,200 per gram of skin in the linea alba along the mid-ventral line and from 1,100 to 16,900 per gram of skin in the region of the wither. In donkeys within the age groups of 1-3, 4-6, and 7-10 years, increasing counts per gram of skin were detected with mean values of 1,083 (range 100-2,400), 1,444 (1,000$2,000)$, and 2,040 (1,330-9,000) microfilariae, respectively (10). The number of $O$. cervicalis microfilariae per 8 or $6 \mathrm{~mm}$ of biopsied skin in ponies and horses ranged from 1 to 21,570 and 8 to 55,600 , respectively (31). In other surveys on horses, microfilarial counts ranged from 18 to 42,446 microfilariae/skin snip (16) and a concentration of 19,770 microfilariae/mg was detected (26).

All four Onchocerca infected jennies appeared to be healthy and clinically normal at the initial clinical examination. This is in agreement with Hussein and El Sammani (5) and Matov et al. (33) in O. cervicalis and O. raillieti infected donkeys, respectively. Matov et al. (33) reported that 12 out of 41 healthy donkeys harbored adults of $O$. cervicalis and eight had microfilariae in their eyes. Hussein and El Sammani (5) found that at postmortem examination, fibrous nodules ranging from $5-10 \mathrm{~mm}$ to $2-5 \mathrm{~cm}$ in diameter, containing degenerated parasites or filled with caseous and calcified necrotic material, were scattered in the ligamentum nuchae. At histological examination, the authors found that live adult worms induced mild inflammation whereas dead worms elicited an intense inflammatory response with the infiltration of large numbers of lymphocytes, macrophages, and fibroblasts intermingled with eosinophils. Moreover, heavy microfilarial skin densities were detected, as mentioned above, and 5 to 15 microfilariae per eye were found, mostly in the anterior chamber. However, despite these findings, none of the 373 infected donkeys had shown clinical signs that could be attributed to the infection, such as poll-evil and fistulous withers, pruritus, blindness or periodic ophthalmia. Likewise, after surveying over 120 horses infected with O. cervicalis, Mellor (34) reported that pathological effects of adult worms on the ligamentum nuchae were of little importance and that no pathological conditions were seen, in either the skin or the eyes, that could be definitely attributed to microfilariae. Matov et al. (33) reported that microfilariae of $O$. cervicalis cannot be considered as the primary cause of periodic ophthalmia in horses. However, other authors have associated O. cervicalis infection in horses with a variety of ocular lesions of the conjunctiva, cornea, uveal tract, lens and retina (35), fistulous withers (36), and dermatitis of the ventral midline and/or limbs, shoulders, thorax, and withers (17). It is thought that microfilariae migrate through the bloodstream reaching the eyelids, cornea, conjunctiva, and uvea where they concentrate and that a marked inflammatory reaction is triggered by immune responses to antigens released by dead and dying microfilariae (1). However, the pathogenic role of $O$. cervicalis still remains uncertain and controversial $(2,37)$ as the infection can be seen in large numbers of horses with or without clinical signs (38). Since we observed the absence of dermatological and ocular signs, we concluded that neither adult worms nor microfilariae appear to cause any clinical evidence in donkeys. It is possible that donkeys may have a higher tolerance than horses to Onchocerca infection.

To the best of our knowledge, only two studies have previously investigated hematological values in donkeys naturally infected with Onchocerca. The results of one of these studies showed that levels of serum total proteins, albumin, and globulins were significantly ( $p<0.05$ ) higher compared to uninfected donkeys. Other variations included an increase in the level of glucose and a lower concentration of serum cholesterol (10). By contrast, in jenny I we found low values of serum total proteins and a mild reduction in albumin along with increased cholesterol. 
However, comparing our findings with those previously reported is not reliable because of the large differences in study design between our report and previous studies. In fact, one study investigated blood biochemical changes following treatment with doramectin against $O$. raillietti microfilariae (9), while another study compared the effects of $O$. raillieti infection on serum total proteins, albumin, glucose, and cholesterol in naturally infected and uninfected donkeys (10). We detected a variety of hematological alterations in one of the jennies (I) with subclinical Onchocerca infection. However, all these alterations were probably due to the blood lipemic status secondary to obstructive colic in a lactating jenny and it is very unlikely that Onchocerca infection plays a key role in this context. Differential white blood cell counts revealed moderate eosinophilia in jenny II while remarkable eosinophilia and basophilia were present in jenny III. The role of eosinophils in parasitic helminth infections in mammals has been widely demonstrated $(39,40)$, including in cases of Onchocerca infection in humans $(41,42)$. The relationship between basophilia and helminth infections has also been well established in humans (43) and several animal models (44). However, it cannot be ruled out that these hematological alterations were caused by concurrent infections with other pathogens. Therefore, further investigations are needed to determine whether most donkeys naturally affected by subclinical infection with Onchocerca may develop eosinophilia and basophilia.

\section{CONCLUSIONS}

Onchocerca microfilariae were incidentally detected in four jennies in Italy. Microfilariae were identified by blood smear, Knott's test, and finally DNA analysis. All the cases were of autochthonous origin because the jennies were born and reared on the same farm located in central Italy and they had never moved outside the farm. The transmission of Onchocerca infection to these animals is linked to their exposure to bites of infected Culicoides, suggesting that vectors in turn could have acquired the infection locally. The reproductive lifespan of Onchocerca volvulus, the etiological agent of river blindness in humans, ranges from 9 to 11 years (45) and adult females can release 700 to 900 microfilariae daily (46). At present, the lifespan of Onchocerca species in donkeys is unknown but we can assume that adult females live for years and likewise release hundreds of microfilariae daily during their lifespan.

\section{REFERENCES}

1. Dagnaw M, Zemene M, Getaneh G, Tibenu S. A review on equine onchocerchiasis. Afr J Basic Appl Sci. (2016) 8:27-33. doi: 10.5829/idosi.ajbas.2016.8.1.22966

2. Melhorn H. Encyclopedia of Parasitology. Berlin; Heidelberg; New York, NY: Springer-Verlag (2008).

3. El Sammani SE, Hussein HS. Onchocerca raillieti: adult location and skin distribution of the microfilaria in Sudanese donkeys. J Helminthol. (1983) 57:355-60. doi: 10.1017/S0022149X0001107X

4. Beveridge I, Gasser R, Sangster N. Horses. In: Beveridge I, Emery D, editors. Australasian Animal Parasites Inside and Out. The Australian Society for Parasitology (2015). p. 826-7.
Insect vectors that are suitable for transmitting the infection are widespread in Italy (20). Since the infection can be easily overlooked, a high proportion of donkeys may be infected with minimal or no clinical or hematological evidence, and may act as clinically asymptomatic carriers. Our report demonstrates the occurrence of Onchocerca in donkeys in Italy. We thus believe that equine practitioners should be aware of our findings and of their implications for equine health. Our findings highlight: (i) the risk of spreading the infection to other susceptible hosts (donkeys, mules, horses, ponies), (ii) the risk of progression in parasitized equines toward clinical disease, and (iii) the need to control the occurrence of Onchocerca infections in the breeding systems of donkeys and horses reared in Italy. The possibility of accidental transmission of $O$. cervicalis to humans has also been reported $(47,48)$. Our report also provides a basis for further studies to determine infection rates in donkeys and horses, to identify the insect vectors that may act as intermediate hosts, and to implement possible prevention and control measures.

\section{DATA AVAILABILITY STATEMENT}

The raw data supporting the conclusions of this article will be made available by the authors, without undue reservation.

\section{ETHICS STATEMENT}

Ethical review was not required as per institutional guidelines/local legislation due to natural infections that occurred spontaneously. Written informed consent was obtained from the owners for the participation of their animals in this study.

\section{AUTHOR CONTRIBUTIONS}

RP performed parasitological examinations and wrote the first draft of the manuscript. GL performed hematological examinations. MS performed all clinical examinations. All the authors critically edited and reviewed the manuscript. All authors contributed to the article and approved the submitted version.

\section{FUNDING}

This study was supported by the University of Pisa (Fondi di Ateneo 2018).

5. Hussein HS, El Sammani SE. Onchocerca raillieti: prevalence and pathology in Sudanese donkeys. J Comp Pathol. (1985) 95:345-51. doi: 10.1016/0021-9975(85)90038-6

6. Lok JB, Cupp EW, Braide EI, Bernardo MJ. Aedes aegypti as a surrogate host for Onchocerca spp. Am J Trop Med Hyg. (1980) 29:382-8. doi: 10.4269/ajtmh.1980.29.382

7. Lia RP, Mutafchiev Y, Veneziano V, Giannelli A, Abramo F, Santoro M, et al. Filarial infection caused by Onchocerca boehmi (Supperer, 1953) in a horse from Italy. Parasitol Res. (2017) 116:191-8. doi: 10.1007/s00436-016-5 277-x

8. Bain O, Muller R, Khamis Y, Guilhon J, Van Veen TS. Onchocerca raillieti sp. (Filarioidea) chez l'Ane domestique en Afrique. J Helminthol. (1976) 50:287-93. doi: 10.1017/S0022149X00026730 
9. Seri HI, ElBashir HM, Elmansoury YHA, Salih MM. Blood biochemical changes in donkeys naturally infected with Onchocerca raillieti: the effect of medication with doramectin. Int J Pharmacol. (2006) 2:530-5. doi: 10.3923/ijp.2006.530.535

10. Elbasheir HM, Adam DA, Anwar AMA. Epidemiology of Onchocerca raillietie in donkeys at Tumbool city and its influence on some serum constituents. Bull Anim Health Prod Afr. (2014) 62:339-43.

11. Zhang Z, Schwartz S, Wagner L, Miller W. Greedy algorithm for aligning DNA sequences. J Comput Biol. (2000) 7:203-14. doi: 10.1089/106652700500 81478

12. Morgulis A, Coulouri G, Raytselis Y, Madden TL, Agarwala R, Schäffer AA. Database indexing for production MegaBLAST searches. Bioinformatics. (2008) 24:1757-64. doi: 10.1093/bioinformatics/btn322

13. Radwan AM, Ahmed NE, Elakabawy LM, Ramadan MY, Elmadawy RS. Prevalence and pathogenesis of some filarial nematodes infecting donkeys in Egypt. Vet World. (2016) 9:888-92. doi: 10.14202/vetworld.2016. 888-892

14. Hasanova RI. Distribution of onchocercosis in horses caused by Onchocerca cervicalis Raillet et Henry 1910, and Onchocerca reticulata Diesing, 1841, in the region of East Caucasus. Rossiiskii Parazitol Z. (2014) 3:48-51 (In Russian).

15. Franck MT, Colombet J, Hugnet C, Ducos de Lahitte J, Desmaizières LM, Delverdier M, et al. Research of skin microfilariae on 160 horses from Poland, France and Spain. Rev Méd Vét. (2006) 157:323-5.

16. Herd RP, Donham JC. Efficacy of ivermectin against Onchocerca cervicalis microfilarial dermatitis in horses. Am J Vet Res. (1983) 49:1102-5.

17. French DD, Klei TM, Foil. CS, Miller RI, Chapman MR, et al. Efficacy of ivermectin in paste and injectable formulations against microfilariae of Onchocerca cervicalis and resolution of associated dermatitis in horses. Am J Vet Res. (1988) 49:1550-4.

18. Monahan CM, Chapman MR, French DD, Klei TR. Efficacy of moxidectin oral gel against Onchocerca cervicalis microfilariae. J Parasitol. (1995) 81:117-8. doi: $10.2307 / 3284021$

19. Mancebo OA, Verdi JH, Bulman GM. Comparative efficacy of moxidectin $2 \%$ equine oral gel and ivermectin 2\% equine oral paste against Onchocerca cervicalis (Railliet and Henry, 1910) microfilariae in horses with naturally acquired infections in Formosa (Argentina). Vet Parasitol. (1997) 73:243-8. doi: 10.1016/S0304-4017(97)00136-2

20. Goffredo M, Meiswinkel R, Federici V, Di Nicola F, Mancini G, Ippoliti C, et al. The 'Culicoides obsoletus group' in Italy: relative abundance, geographic range, and role as vector for Bluetongue virus. Vet Ital. (2016) 52:235-41. doi: 10.12834/VetIt.35.100.1

21. Abou-Rawash AA. Histopathology of nuchal ligament onchocerciasis and microfilarial dermatitis in donkeys in Kafr el-Sheikh governorate. Minufya Vet J. (2002) 2:323-32.

22. Loyd S, Soulsby EJ. Survey of infection with Onchocerca cervicalis in horses in eastern United States. Am J Vet Res. (1978) 39:1962-3.

23. Collins RC. Onchocerciasis of horses in southeastern Louisiana. J Parasitol. (1973) 59:1016-20. doi: 10.2307/3278636

24. Stannard AA, Cello RM. Onchocerca cervicalis infection in horses from the western United States. Am J Vet Res. (1975) 36:1029-31.

25. Schmidt GM, Krehbiel JD, Coley SC, Leid RW. Equine onchocerciasis: lesions in the nuchal ligament of midwestern U.S. horses. Vet Pathol. (1982) 19:16-22. doi: 10.1177/030098588201900104

26. Cummings E, James ER. Prevalence of equine onchocerciasis in southeastern and midwestern United States. J Am Vet Med Assoc. (1985) 186:1202-3.

27. Foil LD, Klei TR, Miller RI, Church GE, Foil CS, French DD, et al. Seasonal changes in density and tissue distribution of Onchocerca cervicalis microfilariae in ponies and related changes in Culicoides variipennis populations in Louisiana. J Parasitol. (1987) 73:320-6. doi: 10.2307/328 2085

28. Abdul-Salam J, al-Taqui M. Seasonal prevalence of Onchocerca-like microfilaria in camels in Kuwait. J Egypt Soc Parasitol. (1995) 25:19-24.

29. Mellor PS. Studies on Onchocerca cervicalis Railliet and Henry 1910: I. Onchocerca cervicalis in British horses. J Helminthol. (1973) 47:97-110. doi: 10.1017/S0022149X00023774
30. Marcoux M, Fréchette JL, Morin M. Infection par Onchocerca cervicalis au Québec: signes cliniques et méthode de diagnostic. Can Vet J. (1977) 18:108-10.

31. Klei TR, Torbert B, Chapman MR, Foil L. Prevalence of Onchocerca cervicalis in equids in the Gulf Coast region. Am J Vet Res. (1984) 45:1646-7.

32. Marques SM, Scroferneker ML. Onchocerca cervicalis in horses from southern Brazil. Trop Anim Health Prod. (2004) 36:633-6. doi: 10.1023/B:TROP.0000042863.83233.78

33. Matov K, Vasilev I, Osikpviski E, Yanchev Y. The importance of microfilariae in the aetiology of periodic ophtalmia in horses. Helminthologia. (1959) 1:257-66.

34. Mellor PS. Studies on Onchocerca cervicalis Railliet and Henry 1910: II Pathology in the horse. J Helminthol. (1973) 147:111-8. doi: 10.1017/S0022149X00023804

35. Cello RM. Ocular onchocerciasis in the horse. Equine Vet J. (1971) 3:148-54 doi: 10.1111/j.2042-3306.1971.tb04460.x

36. Ottley ML, Dallemagne C, Moorhouse DE. Equine onchocerciasis in Queensland and the Northern Territory of Australia. Aust Vet J. (1983) 60:200-3. doi: 10.1111/j.1751-0813.1983.tb09582.x

37. Polley L. Onchocerca in horses from Western Canada and the northwestern United States: an abattoir survey of the prevalence of infection. Can Vet J. (1984) 25:128-9.

38. Moran CT, James ER. Equine ocular pathology ascribed to Onchocerca cervicalis infection: a re-examination. Trop Med Parasitol. (1987) 38:287-8.

39. Behm CA, Ovington KS. The role of eosinophils in parasitic helminth infections: insights from genetically modified mice. Parasitol Today. (2000) 16:202-9. doi: 10.1016/S0169-4758(99)01620-8

40. Klion AD, Nutman TB. The role of eosinophils in host defense against helminth infections. J Allergy Clin Immunol. (2004) 113:30-7. doi: 10.1016/j.jaci.2003.10.050

41. Pearlman E. Immunopathology of onchocerciasis: a role for eosinophils in onchocercal dermatitis and keratitis. Chem Immunol. (1997) 66:26-40. doi: 10.1159/000058664

42. Nfon CK, Makepeace BL, Njongmeta LM, Tanya VN, Bain O, Trees AJ. Eosinophils contribute to killing of adult Onchocerca ochengi within onchocercomata following elimination of Wolbachia. Microb Infect. (2006) 8:2698-705. doi: 10.1016/j.micinf.2006.07.017

43. Voehringer D. The role of basophils in helminth infection. Trends Parasitol. (2009) 25:551-6. doi: 10.1016/j.pt.2009.09.004

44. Mitre E, Nutman TB. Basophils, basophilia, helminth infections. Chem Immunol Allergy. (2006) 90:141-56. doi: 10.1159/000088886

45. Plaisier AP, van Oortmarssen GJ, Remme J, Habbema JD. The reproductive lifespan of Onchocerca volvulus in West African savanna. Acta Trop. (1991) 48:271-84. doi: 10.1016/0001-706X(91)90015-C

46. Schilz-Key H. Observations on the reproductive biology of Onchocerca volvulus. Acta Leidensia. (1990) 59:27-44.

47. Beaver PC, Yoshimura H, Takayasu S, Hashimoto H, Little MD. Zoonotic Onchocerca in a Japanese child. Am J Trop Med Hyg. (1989) 40:298-300. doi: 10.4269/ajtmh.1989.40.298

48. Burr WE Jr, Brown MF, Eberhard ML. Zoonotic Onchocerca (Nematoda: Filarioidea) in the cornea of a Colorado resident. Ophthalmology. (1998) 105:1494-7. doi: 10.1016/S0161-6420(98)98035-6

Conflict of Interest: The authors declare that the research was conducted in the absence of any commercial or financial relationships that could be construed as a potential conflict of interest.

Copyright (c) 2020 Papini, Lubas and Sgorbini. This is an open-access article distributed under the terms of the Creative Commons Attribution License (CC BY). The use, distribution or reproduction in other forums is permitted, provided the original author(s) and the copyright owner(s) are credited and that the original publication in this journal is cited, in accordance with accepted academic practice. No use, distribution or reproduction is permitted which does not comply with these terms. 